\title{
Triagem neurológica de recém-nascidos pré-termo com hemorragias peri e intraventriculares: estudo transversal observacional
}

\author{
Neurological screening of preterm infants with peri- and intra-ventricular \\ hemorrhage: observation transversal study \\ Andréa Vancetto Maglione ${ }^{1}$, Marina Ortega Golin², Roseli Oselka Saccardo Sarni ${ }^{3}$ \\ ${ }^{1}$ Curso de Fisioterapia, Faculdade de Medicina do ABC (FMABC) - Santo André (SP), Brasil. \\ ${ }^{2}$ Disciplina de Fisioterapia Neuropediátrica, FMABC - Santo André (SP), Brasil. \\ ${ }^{3}$ Departamento de Pediatria, FMABC - Santo André (SP), Brasil.
}

DOI: https://dx.doi.org/10.7322/abcshs.v43i1.1162

Introdução: Recém-nascidos pré-termo (RNPT) com menos de 27 semanas de idade gestacional apresentam vulnerabilidade para o desenvolvimento de hemorragias peri e intraventriculares (HPIV), o que pode afetar a mielinização e organização do córtex cerebral, acarretando possíveis prejuízos ao desenvolvimento. Objetivo: Avaliar o comportamento neurológico de RNPT acometido por HPIV com e sem sepse segundo a versão resumida do Método Dubowitz, delimitar a presença de itens desviantes da avaliação e comparar com as respostas obtidas pelo grupo controle (sem HPIV). Métodos: Estudo transversal realizado no Hospital Municipal Universitário de São Bernardo do Campo (SP). Os RNPT foram divididos em três grupos, sendo dois estudos (HPIV e HPIV + sepse) e um controle. Os participantes foram avaliados com idade correspondente ao termo. A versão resumida do método, utilizada como triagem para recém-nascidos de risco neurológico é constituída por 12 itens. As análises foram realizadas segundo pontuações desviantes nestes itens e comparadas com as pontuações esperadas para a normalidade. Os dados obtidos foram comparados na pontuação resumida e na pontuação da versão íntegra. Resultados: A frequência de RNPT com pontuações atípicas foram: $40 \%$ no grupo HPIV + sepse, $10 \%$ no grupo HPIV e $15 \%$ no controle. Conclusão: A HPIV de forma isolada não parece ser um fator significante para presença de itens desviantes no Método Dubowitz resumido. A presença de HPIV em graus mais severos esteve associada à presença de sepse. Os RNPT com HPIV associada à sepse obtiveram pior desempenho neurológico.

Palavras-chave: hemorragia intracranianas; exame neurológico; nascimento prematuro; deficiências do desenvolvimento.
Introduction: Preterm infants with less than 27 weeks of gestacional age present vulnerability for development of periand intraventricular hemorrhage (PIVH). This can affect the myelinization and organization of cerebral cortex, leading to possible developmental impairment. Objective: To evaluate the neurological behavior of preterm infants affected by PIVH with and without sepsis according to the Dubowitz Method summary, to delimit the presence of deviant items of the evaluation and to compare with the results of the control group (without PIVH). Methods: This is a cross-sectional study. The preterm infants were divided in three groups, two study groups (PIVH and PIVH + sepsis), and one control group. The summary version of the Dubowitz Method was used as a neurological screening for the risk in preterm infants. The analysis was performed according to scores with deviation in these items and compared to the expected normal score. Data obtained were compared using the summary score and the full version of the test. Results: The frequency of abnormal scores was: $40 \%$ in the group with PIVH + sepsis, $10 \%$ in the group only with $\mathrm{PIVH}, 15 \%$ in the control group. Conclusion: The PIVH does not seem to be a significant factor for the presence of items with deviation in the summary version of Dubowitz Method. The presence of PIVH in more severe stages was associated with the presence of sepsis. The preterm infants with PIVH with sepsis obtained worse neurological performance in the test.

Keywords: Intracranial hemorrhages; neurologic examination; premature birth; developmental disabilities. 


\section{INTRODUÇÃO}

$\mathrm{O}$ atual aumento de estudos sobre recém-nascidos pré-termo (RNPT) possibilitou o conhecimento de suas peculiaridades e contribuiu para o aumento da sobrevida destes bebês ${ }^{1}$. Porém, com incremento também da incidência de doenças neurológicas, principalmente de hemorragias intracranianas. Isto se deve à vulnerabilidade do sistema nervoso imaturo associado ao déficit na autorregulação vascular desta população ${ }^{2}$.

Os tipos mais frequentes de hemorragias intracranianas são as peri e intraventriculares (HPIV), com incidência, no Brasil, entre 26 e $51 \%{ }^{3}$. A literatura mostra que em RNPT com menos de 27 semanas de idade gestacional, qualquer tipo de HPIV pode afetar a mielinização e organização do córtex cerebral, acarretando possíveis prejuízos ao desenvolvimento ${ }^{4}$. Linder et al. ${ }^{5}$, em estudo com RNPT de baixo peso demonstrou que a sepse precoce pode constituir um fator de risco para o desenvolvimento de HPIV, principalmente em graus elevados.

De maneira geral, os RNPT acometidos por HPIV possuem maiores riscos de desenvolver disfunções do neurodesenvolvimento. Estudo recente mostrou que a presença de HPIV aumentou três vezes o risco de paralisia cerebral em RNPT com extremo baixo peso $(<1000 \mathrm{~g})^{6}$.

Para detectar a presença de HIPV, a ultrassonografia é amplamente utilizada como ferramenta diagnóstica, porém apresenta baixa sensibilidade para hemorragias pequenas ${ }^{7}$. A ressonância nuclear magnética provém informações adicionais, como o tempo e evolução da hemorragia ${ }^{7}$. Os exames neurológicos clínicos são aplicáveis em qualquer ambiente e permitem identificar risco de disfunções neurológicas futuras ${ }^{8}$.

Estudo sobre o emprego associado da avaliação neurológica clínica e da ultrassonografia cerebral neonatal, ambos não invasivos, de baixo custo e rápida aplicação, mostrou alto valor preditivo diagnóstico?. A avaliação clínica pode identificar achados neurológicos em crianças que não apresentavam lesão considerável no exame de imagem ${ }^{10}$.

Há vários métodos de avaliação neurológica neonatal. $\mathrm{O}$ proposto por Dubowitz e Dubowitz, em 1981, possibilita a identificação precoce de incapacidades neurológicas, com alta confiabilidade científica e reconhecimento internacional2,11. É constituído por 34 itens, tem alta sensibilidade para diagnóstico de hemorragias, fácil aplicabilidade e pode ser utilizado por qualquer profissional da saúde ${ }^{12}$. Apesar disso, em uma rotina hospitalar com muitas crianças e escassez de recursos é difícil implantá-lo pela demanda de tempo necessária para sua aplicação. Por esse motivo foi formulada uma versão resumida do teste com 12 itens de fácil interpretação e rápida aplicação ${ }^{13}$. Sua finalidade inicial é triar crianças que necessitam de avaliação neurológica mais detalhada. Aquelas que apresentam dois ou mais itens considerados desviantes são elegíveis para aplicação do Método Dubowitz completo ${ }^{14}$. Foi demonstrado que essa versão resumida é capaz de identificar anormalidades neurológicas em 98,5\% dos RNPT avaliados ${ }^{4}$.

Os primeiros anos de vida constituem o período de maior formação de habilidades primordiais e ganhos funcionais, constituindo o período de maior plasticidade neuronal. Por isso, é de suma importância detectar essas anormalidades no período neonatal para possibilitar a implementação de medidas de intervenção precoce, evitando a instalação de comorbidades permanentes e impactando na qualidade de vida em curto e longo $\operatorname{prazo}^{7,14}$.

No entanto, mesmo perante a inquestionável importância da implementação de uma triagem neurológica neonatal de fácil e rápida aplicação, não são encontrados registros de estudos que analisem a versão resumida do Método Dubowitz em RNPT com HPIV. Dessa forma, pesquisas neste campo podem contribuir para o estabelecimento de sua efetividade em detectar precocemente comprometimentos neurológicos.

Sendo assim, o objetivo do presente estudo foi avaliar o comportamento neurológico de RNPT com HPIV, segundo a versão resumida do Método Dubowitz. Além disso, buscou-se identificar e delimitar a presença de itens desviantes da avaliação, denominados como sinais de alerta; estabelecer o perfil das pontuações dos RNPT com diferentes graus de HPIV; comparar o comportamento neurológico de RNPT com HPIV (grupo estudo) e sem HPIV (grupo controle).

\section{MÉTODOS}

Trata-se de um estudo transversal observacional com 40 RNPT, sendo 10 RNPT com diagnóstico de HPIV pelo ultrassom transfontanela (Grupo Estudo 1), 10 RNPT com diagnóstico de HPIV e de Sepse (Grupo Estudo 2) e 20 RNPT sem estes achados diagnósticos (Grupo Controle). O período de coleta foi de cinco meses. A amostragem não foi probabilística, sendo que foi necessária a inclusão de um grupo de RNPT com diagnóstico de HPIV associada à sepse devido à baixa prevalência de HPIV de forma isolada.

Os participantes do estudo passavam em consulta no Ambulatório Canguru ou estavam internados na Unidade de Terapia Intensiva (UTI) Neonatal ou de Médio Risco do Hospital Municipal Universitário de São Bernardo do Campo /SP, pertencente à rede conveniada da Faculdade de Medicina do ABC (FMABC).

O projeto foi encaminhado ao Comitê de Ética em pesquisa da Faculdade de Medicina do ABC e obteve aprovação em 24 de Fevereiro de 2016 (CAAE: 51905715.3.0000.0082).

Os critérios de inclusão para o Grupo Controle consistiram em: idades gestacionais ao nascimento entre 25 e 36 semanas de gestação; idade correspondente a termo no momento da avaliação (entre 37 e 42 semanas); ausência de registros de asfixia neonatal; apgar do $5^{\circ}$ minuto maior que sete; ultrassom craniano normal; ausência de diagnóstico de sepse ou infecção neonatal; assinatura de termo de consentimento livre e esclarecido pelo responsável.

Os critérios para composição do Grupo Estudo 1 foram: idades gestacionais ao nascimento entre 25 e 36 semanas de gestação; idade correspondente a termo no momento da avaliação (entre 37 e 42 semanas); HPIV diagnosticada pelo ultrassom craniano; 
ausência de registros de asfixia neonatal; apgar do $5^{\circ}$ minuto maior que sete; assinatura de termo de consentimento livre e esclarecido pelo responsável. No caso do Grupo Estudo 2 também deveria haver diagnóstico médico de sepse ou infecção neonatal.

Os critérios de exclusão para ambos os grupos foram: uso de ventilação mecânica ou sedação no momento da avaliação; presença de más formações e infecções do Sistema Nervoso, síndromes congênitas e anóxias.

Os RNPT foram avaliados em um único momento, pela mesma avaliadora.

Para caracterização da população estudada foram coletados os seguintes dados de prontuário:

1. Características maternas e da gestação: realização de pré-natal, número de consultas; uso de tabaco ( $\operatorname{sim} /$ não), álcool (sim/não) e drogas ilícitas (sim/não) durante a gestação; uso de antibióticos ou outros medicamentos; hipertensão arterial, diabetes, infecção urinária e outras doenças durante a gestação; presença de placenta prévia ou ruptura prematura da membrana e tipo de parto.

2. Características do RNPT: sexo, idade gestacional e peso ao nascimento; apgar no primeiro e quinto minutos de vida; intercorrências no período pré, peri e pós natal, complicações respiratórias, cardíacas, icterícia, convulsão, hemorragias intracranianas; infecções neonatais; diagnóstico de HPIV; alterações no ultrassom craniano; uso, tipo e permanência em ventilação mecânica; uso de medicamentos (sim/não/quais).

O diagnóstico e a graduação da HPIV foram embasados pelos resultados do ultrassom craniano conforme critério de Papile que identifica quatro graus de hemorragia conforme sua extensão ${ }^{15}$.

Os RNPT foram classificados de acordo com o peso ao nascimento e o esperado em: pequenos (peso inferior ao percentil 10), adequados (peso entre os percentis 10 e 90 ) ou grandes para a idade gestacional (acima do percentil 90) ${ }^{16}$. A idade gestacional foi estimada através de avaliação clínica pelo método de Capurro ${ }^{17}$ ou pela Escala de New Ballard ${ }^{18}$, que faz parte da rotina dos neonatologistas da unidade hospitalar na qual foi realizada a pesquisa.

As avaliações neurológicas foram realizadas entre as mamadas, nos estágios 4 e 5 de sono e vigília, conforme preconizado por Brazelton ${ }^{19}$. O protocolo de avaliação é a versão resumida do Método Dubowitz, que consiste de 12 itens que avaliam seis dimensões do desenvolvimento: tônus (sete itens), reflexo (um item), movimento (um item), sinal anormal (um item) e comportamento (um item). Há um diagrama com as pontuações e cada item apresenta de três a cinco opções de respostas esperadas, sendo que os itens considerados como alerta são delimitados nas colunas laterais ${ }^{10}$. Os RNPT que apresentaram dois ou mais itens de alerta foram classificados como de risco para disfunções do neurodesenvolvimento ${ }^{8}$. Foi considerado como típico na análise estatística aquele que apresentava menos de 2 itens de alerta e atípico aquele que apresentava 2 ou mas itens de alerta, segundo o Método Dubowitz Resumido. Foi realizada também a análise da avaliação do Método Dubowitz Resumido segundo a pontuação pelo Método Completo, onde cada item possui uma pontuação individual específica esperada para a normalidade ${ }^{20}$.

Para análise dos dados, as variáveis qualitativas foram apresentadas por frequência absoluta e relativa. Para as variáveis quantitativas com não distribuição normal dos dados (Shapiro-Wilk, $\mathrm{p}$-value $<0,05)$ foram adotados mediana e percentis 25 e 75 . Para analisar associação entre os itens desviantes entre os grupos foi utilizado o teste Qui-quadrado. O programa utilizado foi o Stata versão 11.0.

\section{RESULTADOS}

A idade das mães apresentou mediana de 29 anos (variação: 1646). Todas tiveram assistência pré-natal, sendo que a mediana do número de consultas foi 6,5 (variação: 2-12). Apenas uma mãe utilizava drogas ilícitas e álcool durante a gestação, e sete (17,5\%) fumavam. Os medicamentos mais utilizados durante o período gestacional foram Metildopa (12\%) e Ácido Fólico associado ao Sulfato Ferroso (12\%). Em relação às doenças maternas durante a gestação, as mais prevalentes foram: doença hipertensiva da gestação (DHEG) presente em 14 (35\%) mulheres, infecção do trato urinário presente em 10 (25\%), pré-eclâmpsia presente em $5(12,5 \%)$ e restrição do crescimento intrauterino presente em 3 $(7,5 \%)$ mulheres. O tipo de parto predominante foi cesárea (70\%).

Os RNPT avaliados apresentaram mediana de peso ao nascimento de $1.737,5 \mathrm{~g}$ (mínimo 810 e máximo $2.470 \mathrm{~g}$ ) e mediana de idade gestacional de 34,1 semanas (mínimo 27,1 e máximo 36 semanas). Demais características desta população encontram-se na Tabela 1.

Conforme mostra a Tabela 1, as complicações infecciosas estavam presentes em $18 \mathrm{RN}$ (45\%), sendo que a sepse precoce estava presente em cinco (12,5\%), sepse tardia em cinco (12,5\%), sepse presumida em um (2,50\%), infecção neonatal em quatro (10\%) e risco infeccioso em 10 (25\%). O risco infeccioso não foi considerado como critério de exclusão para o Grupo Estudo 1 e para o

Tabela 1: População de recém-nascidos pré-termo avaliados

\begin{tabular}{|l|c|c|}
\hline Feminino & n & $\%$ \\
\hline Masculino & 19 & 47,5 \\
\hline Adequados para a idade gestacional & 21 & 52,5 \\
\hline Pequenos para a idade gestacional & 27 & 67,5 \\
\hline Gemelares & 13 & 32,5 \\
\hline Complicações & 10 & 25 \\
\hline Icterícia & & \\
\hline Respiratórias & 27 & 67,5 \\
\hline Cardíacas & 28 & 70 \\
\hline Convulsões & 10 & 25 \\
\hline Infecciosas & 3 & 7,5 \\
\hline Uso de antibióticos & 18 & 45 \\
\hline Oxigenioterapia e Suporte Ventilatório & 11 & 27,5 \\
\hline CPAP* & & \\
\hline Ventilação Mecânica não invasiva & 10 & 25 \\
\hline Ventilação Mecânica invasiva & 5 & 12,5 \\
\hline Oxigenioterapia & 9 & 22,5 \\
\hline *PAP = continuous positive airway pressure & 14 & 35 \\
\hline
\end{tabular}

${ }^{*} \mathrm{CPAP}=$ continuous positive airway pressure 
Grupo Controle, por não se tratar de um quadro infeccioso devidamente instalado. O Método de determinação da idade gestacional mais utilizado foi o Capurro, em 29 (74,36\%), seguido do New Ballard em 9 (23.08\%). Apenas em um caso foi utilizada a data da última menstruação (2,56\%).

Dos 40 RNPT avaliados, apenas 23 (57.5\%) realizaram o ultrassom transfontanela, por apresentarem suspeita clínica de HPIV. Com relação ao resultado do exame, cinco RNPT $(21,74 \%)$ apresentavam HPIV Grau I Bilateral, um (4,35\%) HPIV Grau I/Grau II, 12 (52,17\%) HPIV Grau II bilateral, um (4,35\%) HPIV Grau II e Grau III, um (4,35\%) HPIV Grau III Bilateral e três (13,04\%) não apresentaram nenhuma alteração.

Em relação aos resultados da avaliação, a Figura 1 mostra o percentual de distribuição da pontuação típica e atípica nos três grupos, demonstrando maior número de pacientes elegíveis para a aplicação do teste completo no grupo HPIV associada à Sepse $(p=0,179)$.

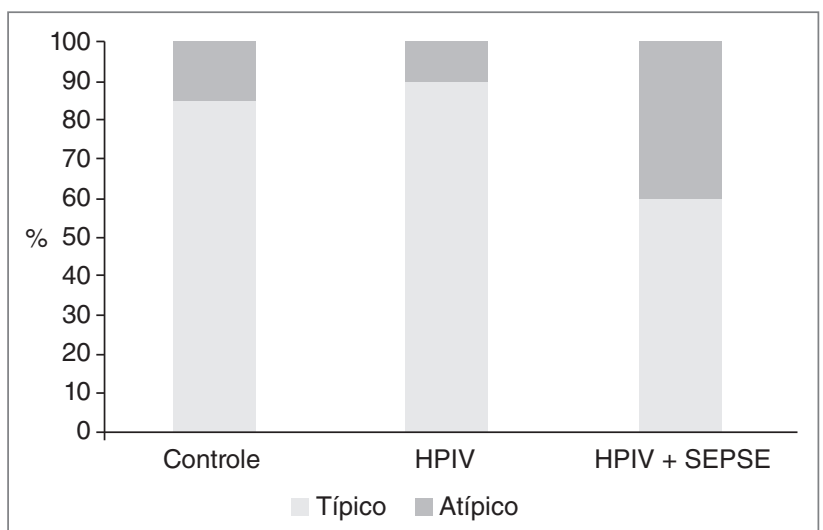

Figura 1: Percentual da distribuição de pontuação típica e atípica, segundo Método Dubowitz Versão Resumida, em recém-nascidos pré-termo com HPIV, HPIV associada à sepse e controle. $p=0,179$.
Na Figura 2 é possível observar o percentual de distribuição da pontuação atípica de cada item do Método Dubowitz Resumido nos três grupos. É possível observar que o grupo com HPIV associada à sepse apresentou maior quantidade de pontuação atípica na maioria dos itens, além de que o item com maior incidência de alteração em todos os grupos foi a Orientação Visual, embora não se tenha comprovada significância estatística em nenhum os casos.

$\mathrm{Na}$ análise da pontuação considerando o Método Dubowitz Completo nota-se que, embora não haja significância estatística, os itens Tração dos Braços, Suspensão Ventral, Movimentação Espontânea (Qualidade) e Orientação Visual foram os mais frequentemente alterados, com percentual geral de classificação atípica de $32,5 \%, 32,5 \%, 35 \%$ e $87,5 \%$ respectivamente.

A Figura 3 mostra o percentual da pontuação típica e atípica no Método Dubowitz Resumido entre os diferentes graus de HPIV. É possível notar que a gravidade da HPIV é diretamente proporcional ao número de itens desviantes $(\mathrm{p}=0,082)$.

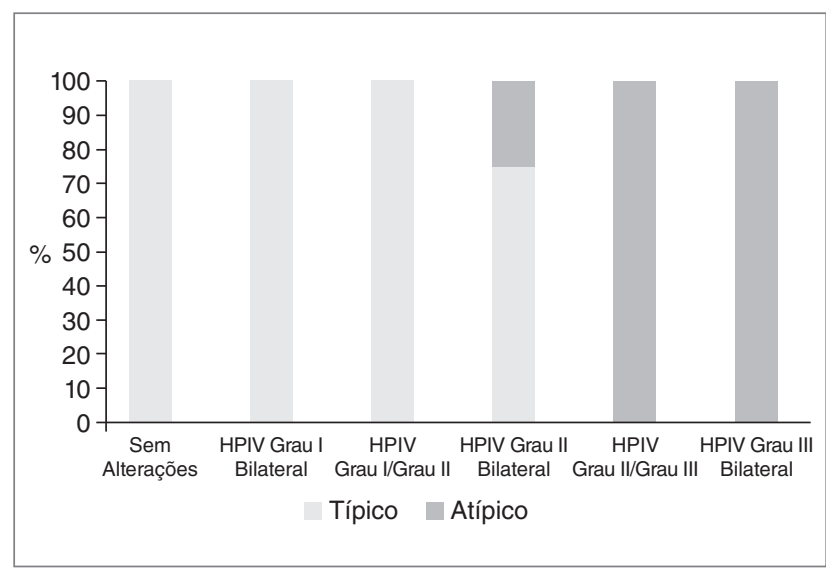

Figura 3: Percentual da pontuação típica e atípica no Método Dubowitz Resumido segundo o resultado do ultrassom transfontanela. $p=0,082$.

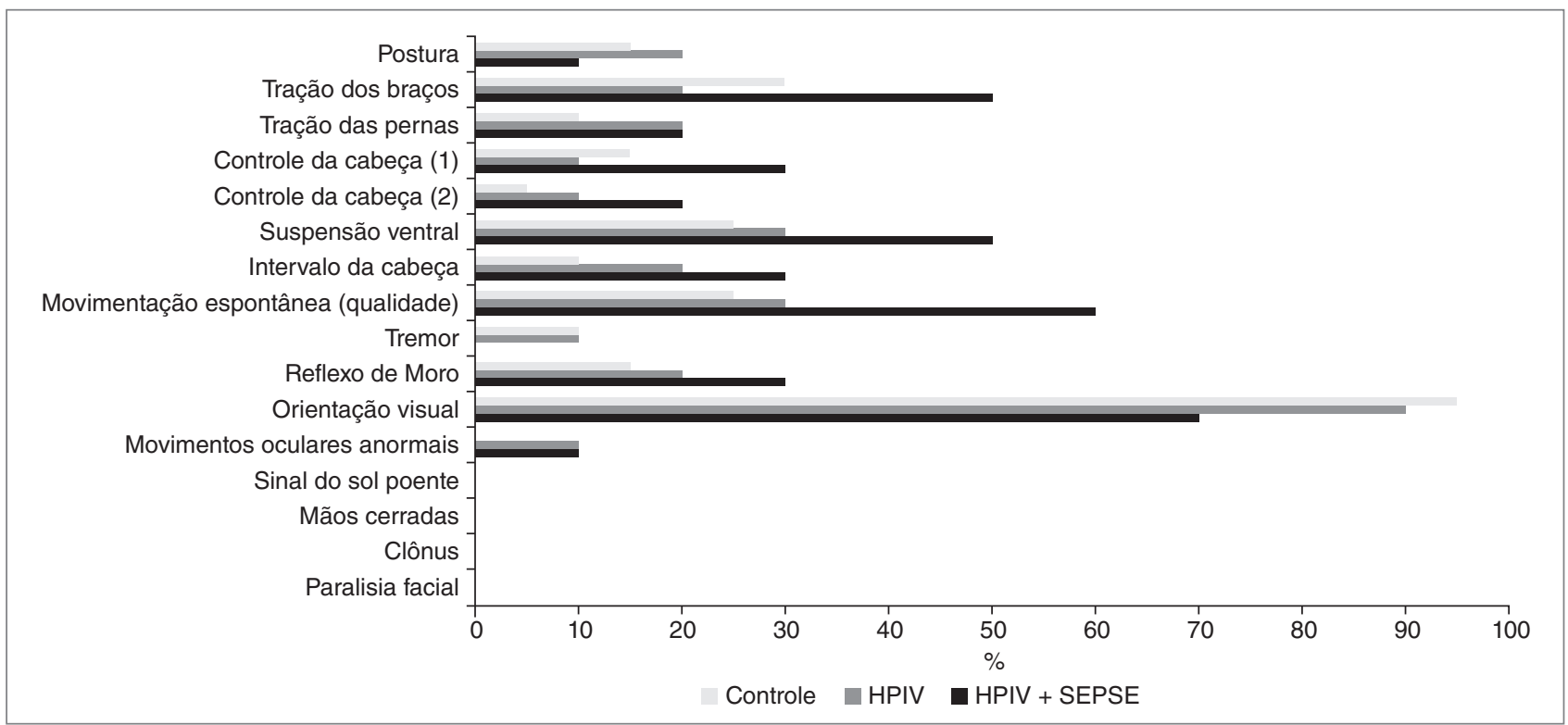

Figura 2: Percentual de distribuição da pontuação atípica de cada item do Método Dubowitz Resumido no grupo controle, HPIV e HPIV associada à sepse. 
O peso ao nascer se mostrou bastante heterogêneo nesta população, por isso esta variável não apresentou distribuição normal dos dados, sendo utilizado teste de o Mann-Whitney com $\mathrm{p}=0,3268$, não demonstrando significância estatística para o pior desempenho no teste. A Tabela 2 mostra a associação dos Itens de Alerta com complicações associadas de ambos os grupos. Foram analisadas as complicações respiratórias, cardíacas, infecciosas e icterícia e observa-se maior associação com as complicações cardíacas e infecciosas, embora não tenha sido comprovada significância estatística.

\section{DISCUSSÃO}

O presente estudo demonstrou que os RNPT com HPIV associada à sepse apresentaram pior desempenho avaliado por meio do Método Dubowitz Resumido.

As hemorragias peri e intraventriculares possuem alta incidência em RNPT devido à imprecisão no controle da pressão intracraniana mediante mudanças na pressão arterial sistêmica, tornando a região periventricular propicia a episódios hemorrágicos ${ }^{2}$. Além disso, essa região é altamente vascularizada por ser fonte de precursores neurais, em especial a partir da vigésima quarta semana gestacional até a idade correspondente de termo ${ }^{21}$. Embora a HPIV seja considerada fator de risco para o neurodesenvolvimento, neste estudo, apenas $10 \%$ dos RNPT com HPIV obtiveram dois ou mais itens de alerta.

O estudo de Grinaboldi et al. ${ }^{22}$, aponta as hemorragias intracranianas como principal fator de risco para desempenho abaixo do esperado pelo Método Dubowitz, no entanto não analisaram RNPT com HPIV de forma isolada. Provavelmente isso não pôde ser visto, neste estudo, pelo perfil do hospital, em que a maioria dos RNPT apresentava associação com outros fatores de risco para o neurodesenvolvimento, em especial quadros infecciosos.

A maioria dos RNPT do grupo estudo 1 tinham alta hospitalar antes de completar 37 semanas de idade corrigida e foram avaliados no Ambulatório de acompanhamento Canguru. No grupo controle, ao contrário, a maioria foi avaliada na UTI ou Berçário Médio Risco, sendo este um possível motivo para que este grupo tenha apresentado maiores proporções de alteração no teste, quando comparados aos RNPT com HPIV. Isto porque o maior tempo de internação pode ser devido à presença de outros fatores de risco, como prematuridade extrema ou baixo peso, ou ser o

Tabela 2: Distribuição de Itens de Alerta segundo as complicações cardíacas, respiratórias, infecciosas e icterícia

\begin{tabular}{l|c|c|c|c|}
\hline & $\mathbf{n}$ & $\begin{array}{c}\text { Menos de 2 } \\
\text { Itens de Alerta }\end{array}$ & $\begin{array}{c}\text { Mais de 2 Itens } \\
\text { de Alerta }\end{array}$ & $\begin{array}{c}\text { Valor } \\
\text { p }\end{array}$ \\
\hline $\begin{array}{l}\text { Complicações } \\
\text { Cardíacas }\end{array}$ & 10 & $6(60 \%)$ & $4(40 \%)$ & 0,068 \\
\hline $\begin{array}{l}\text { Complicações } \\
\text { Respiratórias }\end{array}$ & 28 & $23(82,14 \%)$ & $5(17,86 \%)$ & 0,605 \\
\hline $\begin{array}{l}\text { Icterícia } \\
\text { Complicações }\end{array}$ & 27 & $21(77,78 \%)$ & $6(22,22 \%)$ & 0,613 \\
Infecciosas & 18 & $13(72,22 \%)$ & $5(27,78 \%)$ & 0,266 \\
\hline
\end{tabular}

fator que trouxe maiores complicações clínicas, devido à exposição prolongada ao ambiente hospitalar e uso de medicamentos ou dispositivos de suporte de vida.

Não há estudos utilizando a aplicação da versão resumida do teste proposto por Dubowitz em RNPT com HPIV. No entanto, neste estudo não se observou associação entre a presença de HPIV isolada e pior desempenho neste método.

A severidade das hemorragias peri e intraventriculares pode ser graduada em quatro níveis: grau I, a hemorragia é limitada à matriz germinativa; grau II, estende-se para os ventrículos, porém sem causar dilatação; grau III, evolui para dilatação ventricular e grau IV, quando atinge extensão parenquimal ${ }^{2}$. Os graus III e IV são mais severos e acarretam maiores proporções de sequelas neurológicas ${ }^{21}$. Silva et al. ${ }^{23}$, mostraram que atrasos no desenvolvimento motor de RNPT estavam relacionados com HPIV severas.

O estudo de Palmer et al. ${ }^{24}$, analisou o desenvolvimento a longo prazo de 39 RNPT, comparando um grupo com HPIV e outro sem sinais de hemorragia ao US Transfontanela. Os resultados mostraram maior incidência de anormalidades neurológicas no grupo com dilatação ventricular. Isso condiz com o presente estudo, que demonstrou que quanto maior o grau de HPIV, maior quantidade de itens de alerta encontrados. Possivelmente devido ao pequeno número de RNPT com HPIV Grau III e IV e à associação destes graus de hemorragia à Sepse, este resultado não tenha se mostrado estatisticamente significante.

Bejar et al. ${ }^{25}$, encontraram forte associação entre necrose da substância branca periventricular e quadros infecciosos, o que levanta a hipótese de que a sepse possa estar relacionada à HPIV. Neste estudo é possível observar que a sepse estava presente em muitos casos de HPIV, principalmente naqueles com graus mais severos.

Em estudo de Hentges et al. ${ }^{26}$, com 411 RNPT de muito baixo peso mostrou-se que a sepse neonatal tardia estava associada ao atraso no neurodesenvolvimento nos primeiros dois anos de vida. Isto, provavelmente devido às respostas inflamatórias sistêmicas que danificam a substância branca e aumentam a incidência de comorbidades associadas. Fato que condiz com o presente estudo, no qual $40 \%$ dos RNPT com sepse associada à HPIV apresentaram dois ou mais itens de alerta no Método Dubowitz Resumido, podendo ser classificados como grupo de risco para disfunções neurológicas.

A pontuação de itens desviantes foi proposta por Molteno et al. $^{27} \mathrm{em}$ seu estudo com 100 RNPT de baixo risco e 100 RNPT com achados atípicos no ultrassom transfontanela, preconizando que 2 ou mais itens desviantes, localizados nas laterais do teste, devem ser considerados com $91 \%$ de sensibilidade para detectar alterações do neurodesenvolvimento. No entanto, no atual estudo, um fato chamou a atenção. Muitos RNPT que não apresentavam dois ou mais sinais de alerta apresentavam pontuações que não seriam consideradas como esperadas para a normalidade pela versão completa do Método Dubowitz ${ }^{20}$. Sendo assim, também foram analisadas as pontuações individuais de cada item, como proposto na versão completa. De acordo com essa versão, os itens 
alterados com maior frequência pertenciam às dimensões tônus (tração dos braços e suspensão ventral), comportamento (orientação visual) e movimentos (movimentação espontânea qualidade). Setänen et al. ${ }^{28}$, em estudo combinando o Método Dubowitz à Ressonância Magnética de crânio ou Ultrassom Transfontanela, mostraram que alterações nas dimensões tônus, postura e comportamento se relacionavam com piores resultados nestes exames. E também referem que os itens que mais se relacionam com a Paralisia Cerebral, quando alterados, são postura, orientação visual, tônus flexor e controle da cabeça.

Nesta pesquisa também foi realizada a análise de complicações clínicas, para averiguar se poderiam constituir um item de confusão. Quando as complicações respiratórias, cardíacas e icterícia foram associadas ao desempenho no Método Dubowitz Resumido, não se evidenciou significância estatística. Entre os RNPT com complicações cardíacas, $40 \%$ apresentaram dois ou mais itens de alerta no teste. Entretanto, não se pode afirmar que estas complicações se relacionam a atrasos do neurodesenvolvimento por não ter sido realizada análise deste fator isolado.
Deve-se ressaltar que este estudo contou com algumas limitações metodológicas: amostra de conveniência, presença de complicações associadas, ambiente de análise variado entre ambulatório e unidades de internação, utilização de dados coletados de prontuários.

Os dados encontrados mostram que a presença de HPIV de forma isolada não foi um fator para desvios no Método Dubowitz resumido. Assim, sugerem que, provavelmente, a HPIV não seja um fator de risco associado a atrasos do neurodesenvolvimento sem estar associada a outros fatores de risco. Os RNPT com sepse associada à HPIV obtiveram menor pontuação no Método Dubowitz, corroborando achados na literatura que apontam a sepse como um fator de risco de destaque para atrasos no neurodesenvolvimento. Os graus mais severos de HPIV estavam relacionados à presença de sepse. Possivelmente, este seja um quadro que acarrete em maiores complicações vasculares.

São necessários mais estudos para confirmar se apenas a análise dos itens de alerta, localizados nas laterais do Método Dubowitz resumido, é suficiente para detecção de atrasos no neurodesenvolvimento.

\section{REFERÊNCIAS}

1. Rugolo LMSS. Growth and developmental outcomes of the extremely preterm infant. J Pediatr. 2005; 81(1): 101-110. http://dx.doi.org/10.1590/S0080-62342009000200030

2. Airoldi MJ, Silva SBC, Souza RCT. Avaliação de recém nascidos pré-termo com hemorragia peri-intraventricular e/ou leucomalácia periventricular. Rev Neurociênc. 2009;17(1):24-9.

3. Marba STM, Caldas JPS, Vinagre LEF, Pessoto MA. Incidência de hemorragia peri-intraventricular em recém-nascidos de muito baixo peso: análise de 15 anos. J Pediatr. 2011;87(6):505-11. http://dx.doi.org/10.1590/S0021-75572011000600008

4. Payne AH, Hintz SR, Hibbs AM, Walsh MC, Vohr BR, Bann CM, et al. Neurodevelopmental outcomes of extremely low-gestationalage neonates with low-grade periventricular-intraventricular hemorrhage. Jama Pediatr. 2013;167(5):451-9.

http://dx.doi.org/10.1001/jamapediatrics.2013.866

5. Linder N, Haskin O, Levit O, Klinger G, Prince T, Naor N, Turner P, Karmazyn B, Sirota L. Risk factors for intraventricular hemorrhage in very low birth weight premature infants: a retrospective casecontrol study. Pediatrics. 2003;111(5):e590-e595. https://doi.org/10.1542/peds.111.5.e590

6. Cunha M, Bettencourt A, Almeida A, Mimoso G, Soares P, Tomé T. O recém nascido de extremo baixo peso. Estado aos 2-3 anos. Resultados do Registo Nacional de Muito Baixo Peso de 2005 e 2006. Acta Pediatr Port. 2013;44(1):1-8.

7. Kutuk MS, Yikilmaz A, Ozgun MT, Dolanbay M, Canpolat M, Uludag S, et al. Prenatal diagnosis and postnatal outcome of fetal intracranial hemorrhage. Childs Nerv Syst. 2014;30(3):411-8. http://dx.doi.org/10.1007/s00381-013-2243-0

8. Wusthoff CJ. How to use: the neonatal neurological examination. Arch Dis Child Educ Pract Ed. 2013;98(4):148-53. http://dx.doi.org/10.1136/archdischild-2013-303640

9. Pedroso FS, Rotta NT. Neurological examination in the healthy term newborn. Arq Neuropsiquiatr. 2003;61(2A):165-9. http://dx.doi.org/10.1590/S0004-282X2003000200001
10. Romeo DM, Ricci D, van Haastert IC, Vries LS, Haataja L, Brogna $C$, et al. neurologic assessment tool for screening preterm infants at term age. J Pediatr. 2012;161(6):1166-8. http://dx.doi.org/10.1016/j.jpeds.2012.07.037

11. Nascimento KK, Casagrande GMA, Golin MO. Avaliação neurológica de recém-nascidos a termo de baixo risco pelo Método Dubowitz. Arq Bras Ciênc Saúde. 2011;36(3):134-9 http://dx.doi.org/10.7322/abcs.v36i3.51

12. Golin MO, Souza FIS, Sarni ROS. Avaliação neurológica pelo método Dubowitz em recém-nascidos prematuros com idade corrigida de termo comparada a de nascidos a termo. Rev Paul Pediatr. 2009;27(4):402-9. http://dx.doi.org/10.1590/S0103-05822009000400009

13. Mercuri E, Ricci D, Pane M, Baranello G. The neurological examination of the newborn baby. Early Hum Dev. 2005;81912):947-56. http://dx.doi.org/10.1016/j.earlhumdev.2005.10.007

14. Fernandes LV, Goulart AL, Santos AMN, Barros MCM, Guerra CC Kopelman BI. Avaliação do neurodesenvolvimento de prematuros de muito baixo peso ao nascer entre 18 e 24 meses de idade corrigida pelas escalas Bayley III. J Pediatr. 2012;88(6):471-8. http://dx.doi.org/10.1590/S0021-75572012000600005

15. Papile L-A, Burstein J, Burstein R, Kofller $\mathrm{H}$. Incidence and evolution of subependymal and intraventricular hemorrhage: a study of infants with birth weights less than 1500 g. J Pediatr. 1978; 92:529-34. https://doi.org/10.1016/s0022-3476(78)80282-0

16. Battaglia FC, Lubchenco LO. A practical classification of newborn infants by weight and gestational age. J Pediatr. 1967;71(2):159-63. http://dx.doi.org/10.1016/S0022-3476(67)80066-0

17. Capurro H, Konichezky S, Fonseca D, Caldeyro-Barcia R. A simplified method for diagnosis of gestational age in the newborn infant. J Pediatr. 1978;93(1):120-2.

http://dx.doi.org/10.1016/S0022-3476(78)80621-0

18. Ballard JL, Khoury JC, Wedig K, Wang L, Eilers-Walsman BL, Lipp R. New Ballard Score, expanded to include extremely premature infants. J Pediatr. 1991:119(3):417-23. http://dx.doi.org/10.1016/S0022-3476(05)82056-6 
19. Brazelton TB. Neonatal Behavioral Assessment Scale. Clin Dev Med. 1973;(50):48-54.

20. Dubowitz L, Mercuri E, Dubowitz V. An optimality score for the neurologic examination of the term newborn. J Pediatr. 1998;133(3):406-16.

http://dx.doi.org/10.1016/S0022-3476(98)70279-3

21. Bouz P, Zouros A, Taha A, Sadanan V. Neonatal Intracerebral Hemorrhage: Mechanisms, Managements, and the Outcomes. Transl Stroke Res. 2012;3(Supl. 1):S6-9. http://dx.doi.org/10.1007/s12975-012-0180-y

22. Grinaboldi A, Hinnig P, Moura SPS, Golin MO. Avaliação neurológica de recém-nascidos pré-termo: correlação com fatores de risco neonatais. Rev Neurocienc. 2015;23(2):267-74. http://dx.doi.org/10.4181/RNC.2015.23.02.1017.8p

23. Silva AJ, Neves LAT, Frônio JS, Ribeiro LC. Factors related to motor developmental delay of newborns. J Hum Growth Dev. 2014;24(3):320-7.

http://dx.doi.org/10.7322/jhgd.88970

24. Palmer P, Dubowitz LMS, Levene MI, Dubowitz V. Developmental and neurological progress of preterm infants with intraventricular haemorrhage and ventricular dilatation. Arch Dis Child. 1982;57(10):748-53.

25. Bejar R, Wozniak P, Allard M, Benirschke K, Vaucher Y, Coen R, et al. Antenatal origin of neurologic damage in newborn infants: I. Preterm infants. Am J Obstet Gynecol. 1988;159(2):357-63. http://dx.doi.org/10.1016/S0002-9378(88)80084-X

26. Hentges CR, Silveira RC, Procianoy RS, Carvalho CG, Filipouski GR, Fuentefria RN, et al. Association of late-onset neonatal sepsis with late neurodevelopment in the first two years of life of preterm infants with very low birth weight. J Pediatr. 2014;90(1):50-7. http://dx.doi.org/10.1016/j.jped.2013.10.002

27. Molteno C, Grosz P, Wallace P, Jones M. Neurological examination of the preterm and fullterm infant at risk for developmental disabilities using the Dubowitz Neurological Assessment. Early Hum Dev. 1995;41(3):167-76.

http://dx.doi.org/10.1016/0378-3782(95)01621-9

28. Setänen S, Lahti K, Lehtonen L, Parkkola R, Maunu J, Saarinen $\mathrm{K}$, et al. Neurological examination combined with brain MRI or cranial US improves prediction of neurological outcome in preterm infants. Early Hum Dev. 2014;90(12):851-6. http://dx.doi.org/10.1016/j.earlhumdev.2014.09.007 